\title{
Financiamiento de la educación superior: el panorama mexicano
}

\author{
Cynthia Paola Fuentes Hernández
}

\begin{abstract}
Resumen
La ausencia de una política de financiamiento permite poca claridad, direccionalidad, pertinencia y certidumbre hacia el quehacer de las instituciones universitarias. Ello ha traído consigo un sistema de prueba y error para este nivel educativo. Este artículo tiene como objetivo principal discutir algunas problemáticas políticas, sociales y económicas que giran en torno a la educación superior en México. Para ello, se hace una revisión bibliográfica exhaustiva en los aspectos modernos que se han impuesto de manera generalizada en la educación terciaria.
\end{abstract}

Palabras clave: política, financiamiento, educación superior.

\section{FINANCING HIGHER EDUCATION: the MEXICAN PANORAMA}

\begin{abstract}
The absence of a financing policy allows little clarity, directionality, relevance and certainty towards the work of university institutions; this has caused a trial and error system for this educational level. The main objective of this article is to discuss some political, social and economic problems that revolve around higher education in Mexico. For this, an exhaustive bibliographic review is made on the modern aspects that have been imposed in a generalized way in tertiary education.
\end{abstract}

Keywords: policy, financing, higher education. 
"Financiamiento de la educación superior: el panorama mexicano"

Cynthia Paola Fuentes Hernández

Vol. 22, Núm. 5, septiembre-octubre 2021

Revista Digital Universitaria

\section{Cynthia Paola Fuentes Hernández}

paola.fuentes@alumnos.udg.mx orcid.org/0000-0002-3232-8583

Doctorante en Gestión de la Educación Superior, Universidad de Guadalajara. Becaria del Consejo Nacional de Ciencia y Tecnología (CONACYT). Se ha desempeñado como profesora universitaria y pertenece a la Red de Estudios Sobre Instituciones Educativas (RESIEDU). Sus contribuciones y producción del conocimiento se dirigen hacia los procesos de gestión en la educación superior.

\section{Introducción}

En México no se cuenta con una política de financiamiento para las instituciones de la educación superior, por lo que estos organismos han tratado de responder por sí mismos ante los diversos retos económicos, políticos, institucionales y sociales. En ese sentido, la actual crisis sanitaria ocasionada por la covid-19 ha incrementado aún más las complejidades para este nivel educativo.

De acuerdo con Salmi (2018), el éxito de cualquier país para potencializar su sistema de educación terciaria depende, en mayor parte, de la disponibilidad de recursos financieros suficientes, así como de la capacidad para instaurar los métodos y procedimientos en el uso de la asignación de los bienes. Una estrategia de financiamiento sostenible requiere de la incorporación de marcos normativos y regulatorios que indiquen las formas de operacionalización para las instituciones universitarias. Se deben establecer las condiciones de los actores, los atributos de las instancias involucradas, el grado de flexibilidad en la recaudación de fondos y, de igual manera, permitir la movilización de recursos bajo criterios de transparencia y rendición de cuentas.

Para el desarrollo de este artículo se hace una revisión bibliográfica en torno al financiamiento de la educación superior, considerando los elementos de carácter político, social y económico. El objetivo principal radica en reconocer la necesidad de una política para el financiamiento de este nivel de estudios, que permita solucionar y atender de manera eficiente los aspectos de modernidad que le han sido impuestos y su deficiencia al interior de las instituciones. 


\section{La educación superior, distante de ser un bien público}

La discursiva de los organismos internacionales - como la unesco y el Comité de las Naciones Unidas - ha posicionado a la educación como un bien público. Si bien, la intencionalidad en los níveles básico y medio superior se dirige a la adquisición de conocimientos generales contribuyendo al desarrollo personal e individual; la educación superior va más allá, ya que mantiene los denominados beneficios colectivos que impactan en la sociedad y al mercado laboral (La educación como bien público, 2014). Son las universidades las únicas con capacidad para generar fuerza de trabajo y producción de nuevos saberes, asî como los mecanismos de acceso al conocimiento. Esta educación, como bien público, atiende dos criterios básicos: la no rivalidad y la no exclusión (Daviet, 2016; Marginson, 2016). Sin embargo, estos criterios se fracturan a la hora de distribuir los recursos económicos en las universidades, además de que existen filtros de selección para el ingreso y admisión de estudiantes, por lo que no es un espacio de acceso abierto.

De tal manera, las instituciones universitarias se han visto forzadas a diversificar sus fuentes de recursos económicos mediante los costos compartidos, el financiamiento privado y el cobro de matrícula, utilizando la extensión universitaria con la venta o renta de servicios (Salmi, 2017). Las estrategias que se han adoptado para regular y controlar la distribución del financiamiento, se basan en esquemas meritorios y en el cumplimiento de criterios de calidad, propios de la evaluación educativa (Brunner, 2013). Con ello se ha generado una brecha de desigualdad entre las instituciones universitarias, ya que aquellas mejor posicionadas logran ganar legitimidad y pueden acceder a mayores recursos de financiamiento, mientras que otras no posicionadas, seguirán obteniendo el recurso básico de operación.

"El problema de la calidad va de la mano de la masificación en un contexto de restricciones financieras" (Licandro y Yepes Chisco, 2018, p. 3), por lo que aquellas instituciones que cuentan sólo con el recurso básico difícilmente podrán aspirar a mejorar en indicadores de calidad, en las condiciones contractuales para sus colaboradores, y en su inserción en materia de ciencia y tecnología. Por estos aspectos, la educación superior se encuentra distante de ser un bien público, aunque por otra parte, mantiene funciones, atribuciones y elementos de un bien común, que posiblemente su reconocimiento sólo se encuentre en transición.

\section{Factores transversales en el financiamiento de la educación superior}

En medio de la crisis sanitaria por la covid-19 se hizo evidente que las universidades no son un espacio de prioridad en el contexto mexicano, ya que a más de un año de iniciar la pandemia, los centros educativos permanecen cerrados y no cuentan con un plan estratégico para su reactivación. 
"Financiamiento de la educación superior: el panorama mexicano"

Cynthia Paola Fuentes Hernández

Vol. 22, Núm. 5, septiembre-octubre 2021

Revista Digital Universitaria

Si bien, los conflictos financieros situados al interior de las instituciones no emergieron con la pandemia, éstos se han complejizado. En este sentido, existen diversos factores económicos, políticos, institucionales, y sociales que demuestran la falta de compromiso normativo por parte del Estado hacia las universidades en relación a los subsidios gubernamentales.

\section{a. Factores económicos}

Hay en México tres períodos presidenciales con mayor representatividad en la instauración de mecanismos para la regulación de los recursos económicos, dirigidos a las instituciones de educación superior.

El primero, de 1982 a 1988, es el de Miguel de la Madrid, donde se comenzaron a vislumbrar aspectos de evaluación para acceder al ejercicio presupuestal. En la década perdida de 1980, la política de gasto gubernamental hacia las universidades tuvo las disminuciones más significativas en esa administración (Hernández, 1998). Pasaron de ser un sector privilegiado a uno castigado (Acosta, 2002; Murayama, 2009).

El segundo período es durante el gobierno de Carlos Salinas de Gortari (1988-1994), en el que la firma del Tratado de Libre Comercio trajo consigo mayor impulso en el desarrollo tecnológico, surgieron instituciones como el Consejo Nacional de Evaluación, los Comités Interinstitucionales para la Evaluación de la Educación Superior A.C., el Centro Nacional de Evaluación para la Educación Superior, y al interior del Consejo Nacional de Ciencia y Tecnología se instauró el Sistema Nacional de Investigadores y el Programa Nacional de Posgrados de Calidad (Canales, 2011). La economía creció 3.4\%, lo que trajo inversión al desarrollo social y a la educación. Sin embargo, apareció el financiamiento privado con su propia misión, haciendo posible la asociación público-privado. De tal modo, pueden distinguirse dos modelos para el otorgamiento de recursos económicos: el tradicional o convencional y la nueva administración pública o emergente (ver tabla 1).

\begin{tabular}{|c|l|}
\hline \multicolumn{1}{|c|}{ Sistema } & \multicolumn{1}{c|}{ Elementos } \\
\hline \multirow{2}{*}{ Tradicional o convencional } & Enfoque asistencial \\
& Financiación pública \\
& Negociación para definir y asignar recursos \\
& Nivel de tasas bajo \\
& Sin préstamos para alumnos \\
& Educación de tiempo completo \\
\hline \multirow{2}{*}{ Nueva administración pública o emergente } & Enfoque mercantil \\
& $\begin{array}{l}\text { Financiación privada } \\
\text { Nivel de tasas alto } \\
\text { Opción de préstamos para alumnos } \\
\\
\text { Educación de tiempo parcial y a distancia }\end{array}$ \\
\hline
\end{tabular}

En el país se han desarrollado modelos de financiamiento mixtos, por lo que las autoridades al interior de las universidades han tenido que diversificar sus fuentes económicas para volverse más competitivas y acceder a los estímulos extraordinarios. 
El último período con impacto en los mecanismos de financiamiento para las universidades es el comprendido a partir del 2018 a la fecha, representado por el mandatario Andrés Manuel López Obrador. De acuerdo con la Organización para la Cooperación y el Desarrollo Económico (OCDE), México es uno de los países con menos inversiones en la educación terciaria (2020); aunado a ello, sin haber propuesto acciones mediáticas o parámetros que pudieran regular la operacionalización de programas con fondos extraordinarios, en el año 2020 se extinguieron 109 fideicomisos, incluyendo los relacionados a la investigación científica y tecnológica.

Sin embargo, una acción acertada del gobierno actual ha sido la Ley General de Educación Superior (LGES), publicada en el Diario Oficial de la Federación (DOF) el 20 de abril de 2021. Por un lado, es un gran paso en materia de los procesos legislativos, en el reconocimiento de la autonomía universitaria y la libertad de cátedra; aunque, por otra
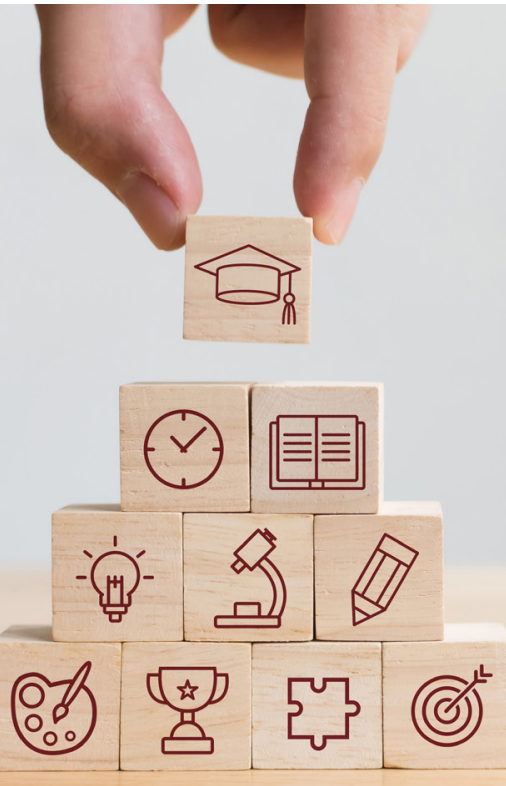

parte, en lo que refiere al financiamiento, ahondan vacíos de operacionalización, así como de los compromisos por parte del Estado para ofrecer gratuidad y cobertura en este nivel.

Con base a estos períodos, se puede establecer que el financiamiento de la educación superior ha estado sujeto a los cambios en la economía mexicana. Una política para el financiamiento de la educación superior puede dirigir las estrategias y acciones para la obtención de recursos ordinarios y extraordinarios; pero también indicar los mecanismos de transparencia y rendición de cuentas referente a los recursos propios.

\section{b. Políticos - institucionales}

La cobertura, calidad, investigación e internacionalización son algunos de los aspectos que han salido a relucir dentro de la modernización a la educación superior, estos elementos son óptimos para negociar recursos ordinarios. Sin embargo, las instituciones han hecho, han entendido y han realizado estrategias para atender de manera general estos aspectos, de acuerdo a sus posibilidades.

La cobertura sigue siendo un reto para las autoridades institucionales y gubernamentales, ya que el aumento en la atención de estudiantes universitarios requiere de particularidades ligadas directamente al financiamiento. La oCDE indica que México es uno de los países que menos aporta en el gasto por estudiante: sólo invirtió 6,586 dólares por alumno, es decir, 9,741 dólares menos que el promedio de los países miembros. Además, de los jóvenes entre 25 a 34 años 
de edad, solamente 24\% obtuvo un título universitario en 2019, mientras que el promedio de los países miembros es de 45\% (2020). De no establecerse acciones financieras para abordar la cobertura, ésta seguirá siendo un reto nacional. En este sentido, es urgente abrir espacios de debate que posibiliten estrategias para la diversificación de los recursos financieros, además de permitir el diálogo referente a la redistribución del pago en matrículas y la distribución de costos.

Referente a la calidad, en México las acciones no han sido del todo favorables para las universidades, de seguir contando con un presupuesto ordinario, las instituciones no podrán atender los parámetros métricos que se establecen en las evaluaciones propias de la calidad.

Un instrumento que se utiliza para medir la calidad de la educación terciaria a nivel global son los rankings, que logran identificar y posicionar a las mejores universidades; las cuales de manera significativa contribuyen a la generación y producción del conocimiento mediante la investigación, al tratar de formar egresados sobresalientes para el mercado laboral global (Salmi, 2017). Es así como las acciones alusivas a la investigación se han venido posicionando en las instituciones de educación superior; de tal forma, que los indicadores valorativos en México favorecen a las universidades que cuenten con académicos investigadores adscritos al Sistema Nacional de Investigadores (SNI).

La contraparte relacionada a este elemento tiene que ver con la escasa relación entre las evidencias investigativas y la toma de decisiones en la política pública, lo que Teresa Bracho (2010) denomina política basada en evidencia. Pareciera que los aspectos de investigación sólo contribuyen a cumplir con las estructuras normativas para la obtención de estímulos y recompensas.

Finalmente, otro elemento instaurado en las instituciones es la internacionalización, cuyo principal limitante es el financiamiento, aunque le siguen otros obstáculos como el manejo de un segundo idioma, complejidades administrativas y burocráticas, falta de estrategias o planes para seguir los procesos, el poco reconocimiento de estudios y transferencia de créditos académicos, entre otras (Gacel y Rodríguez, 2018). Por lo que, las universidades que cuentan con recursos para hacer este tipo de actividades, habrán de impulsar estrategias como la internacionalización en casa, o la internacionalización del currículum. Asimismo, podrían fortalecer, en el profesorado y en el estudiantado, la adopción de otros idiomas y la familiarización de la lectura en otra lengua.

Entonces, para poder atender estos aspectos de modernidad, se requiere la construcción de una política de financiamiento en la educación superior, que garantice la suficiencia del recurso, la certeza de operatividad, que logre permitir un equilibrio equitativo entre las instituciones, además de fortalecer la transparencia y la rendición de cuentas. 


\section{c. Sociales}

Los factores sociales que permean la educación superior se direccionan hacia la inclusión, equidad y responsabilidad social. En la reciente LGES (2021), se promueven estos factores, así como la obligatoriedad y la gratuidad de forma gradual para este nivel; además, se establece que la formación universitaria de individuos debe abarcar los distintos campos de la ciencia, la tecnología y las humanidades. Por ello, las autoridades universitarias habrán de incorporar en sus planes estos ejes, para atender lo establecido en la normativa federal. Además, habrán de generar y propiciar estrategias orientadas a a los denominados grupos vulnerables.

En busca de incrementar la inclusión, se consideran por lo general actividades de infraestructuras, - uso de elevadores, rampas, sanitarios, entre otros-; sin embargo, se deja fuera el conocimiento de estos grupos sobre las condiciones y características de los estudiantes, qué tipo de apoyo es el que requieren, con cuánto recurso dispone cada universidad para apoyos de esta índole, etcétera. Se necesita de una mirada multidimensional para poder atender de manera pertinente este eje. El fin de la inclusión, es no apartar a los aspirantes del nivel superior, y en ello radica el quehacer de los gestores en la educación, centrarse en generar apoyos previos, durante y posteriores a la vida universitaria.

Con relación a la equidad, la mayoría de los países en América Latina mantienen en sus políticas un enfoque tradicional, con un énfasis en la ayuda financiera como mecanismo principal, a excepción de Chile (Brunner, 2013); en lugar de promover intervenciones para mejorar las posibilidades de éxito de los estudiantes universitarios en contextos desfavorecidos (Salmi, 2018). Un estudio realizado por Jamil Salmi en el 2018 comparó las políticas de 71 países en todos los continentes, y se incluyen las relacionadas al fomento de equidad en la educación superior. Los países se diferencian en cuatro categorías:

1. Emergente. Nueve de los países cuentan en sus políticas de equidad con principios y objetivos, pero no han tenido intervenciones concretas.

2. En desarrollo. 33 países cuentan con una estrategia para promover la equidad, pero no definen políticas, programas, e inversiones.

3. Establecido. 23 de los países tienen una estrategia para promover la equidad mediante sus políticas, así como los mecanismos para cumplirlas.

4. Avanzado. Sólo seis países han formulado y establecido una estrategia integral para promover los aspectos de equidad.

Poco más de 59\% de los países se encuentran como emergentes o en desarrollo, por lo que las acciones aún son necesarias en materia de este tema. Además, es necesario incorporar estrategias de equidad para víctimas de violencia sexual, para miembros de la comunidad LGBT+, jóvenes húerfanos, refugiados, y estudiantes con cuidados médicos (Salmi, 2018). 
Finalmente, la responsabilidad social es otro eje que recientemente necesita atención por parte de las instituciones de educación superior. La universidad requiere fortalecer su compromiso moral con el desarrollo humano, retomar las asignaturas de ética y valores para la formación de los estudiantes, así como realizar un análisis de sostenibilidad para autoevaluarse (Vallaeys, 2018).

Entonces, con relación a los ejes de inclusión, equidad y responsabilidad social, éstos permanecen en la discursiva de las autoridades gubernamentales, pero aún faltan estrategias precisas para avanzar en dichos aspectos. Si bien, la LGES mantiene un fuerte discurso y orientación a favorecer a los grupos vulnerables, por otro lado, hay ausencia en cuanto a las metas, los objetivos y los criterios de medición para abordar estos ejes, por lo que sigue quedando ambigua, subjetiva e incierta su encomienda.

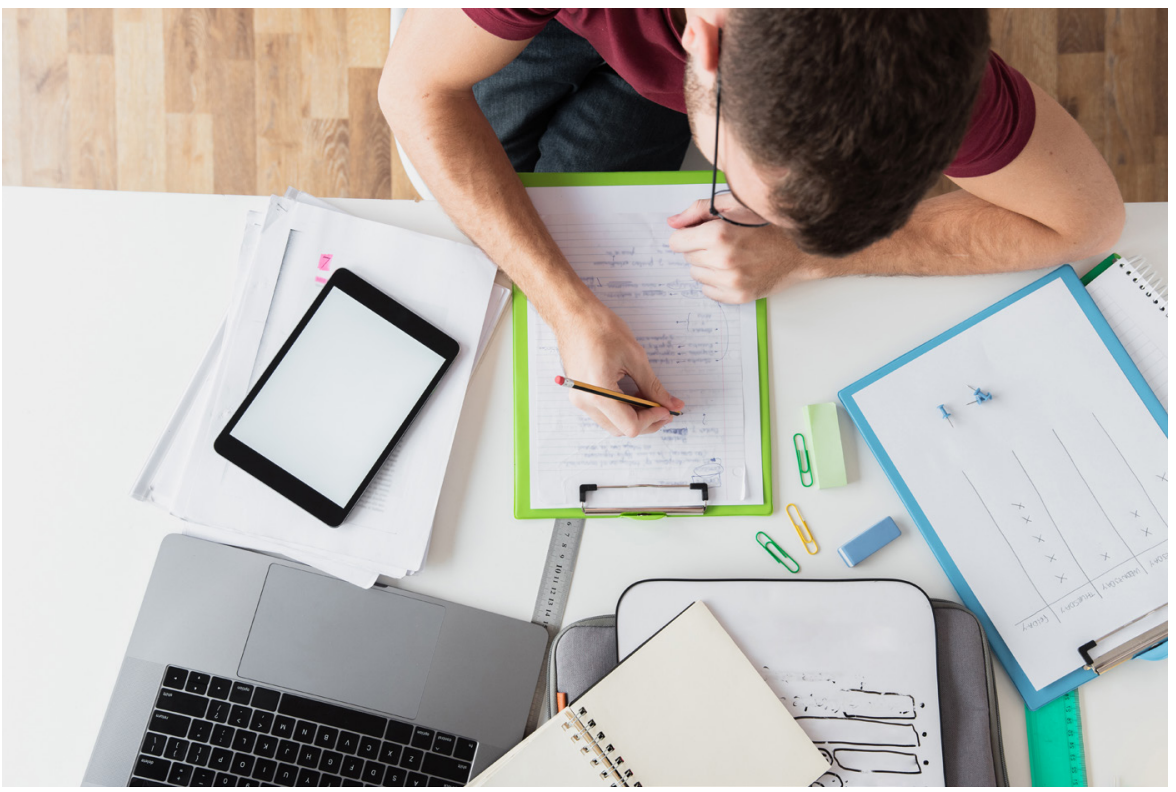

\section{Conclusiones}

El gobierno mexicano no ha podido establecer ni direccionar mecanismos de capital para la educación superior pública, lo que ha imposibilitado una paridad evolutiva sobre el tema, y por supuesto un modelo económico progresivo para las instituciones públicas. La trayectoria que ha tenido el financiamiento de este nivel de estudios mantiene sus matices en las etapas económicas y políticas de gobierno.

Los cambios en la modernidad impulsan a las instituciones a buscar nuevas estrategias para la adquisición de recursos económicos. De acuerdo con Salmi (2018), existe otra opción para los países en desarrollo sin necesidad de centrarse en el financiamiento, tales como: reformar los procedimientos de admisión, establecer instituciones en áreas remotas, fortalecer la educación a distancia, ejercer programas de retención, considerar el apoyo de donantes, entre otras.

Desafortunadamente, el nuevo escenario pandémico pone en riesgo de clausurar las universidades en las cuales su única fuente de financiamiento sea la matrícula; y aquellas que permanezcan abiertas tendrán que asumir riesgos en aspectos de calidad educativa, considerar la eliminación de programas educativos, reducciones en la investigación e internacionacionalización, restricciones en mejoras de infraestructura, e incluso posibles despidos al profesorado de tiempo parcial. 
"Financiamiento de la educación superior: el panorama mexicano"

Cynthia Paola Fuentes Hernández

Vol. 22, Núm. 5, septiembre-octubre 2021

Revista Digital Universitaria

Hace falta mantener una mirada multidimensional que trascienda de la obtención del financiamiento para las universidades, hacia los beneficios tangibles e intangibles que estas pueden propiciar. Direccionar a la educación como uno de los derechos de primera generación permitirá encauzar todos sus elementos y particularidades —incluyendo el financiamiento-, para progresar y mejorar las condiciones en que se provee.

\section{Referencias}

* Acosta, A. (2002). El Neointervencionalismo estatal en la educación superior en América Latina. Revista Sociológica, 17(49), 43-72. https://www.redalyc.org/ pdf/3050/305026562003.pdf

* Bracho, T. (2010). Políticas públicas basadas en la evidencia. La política pública como acción informada y objeto de investigación. En M. Merino y G. Cejudo (comps.), Problemas, decisiones, soluciones. Enfoques de política pública (pp. 291319). FCE/CIDE.

* Brunner, J. (2013). Financiamiento de la educación superior en América Latina: Viejas y nuevas prácticas. Revista Latin American Studies Association Forum, 44(2), 15-17. https://cutt.ly/6WZMQzp

* Canales, A. (2011). La política científica y tecnológica en México. El impulso contingente en el período 1982-2006. Ed. Miguel Ángel Porrúa.

* Daviet, B. (2016). Revisar el principio de la educación como bien público. En Investigación y prospectiva en educación [Documentos de trabajo]. unEsco. https:// core.ac.uk/download/pdf/143615352.pdf

* Gacel, J. y Rodríguez, S. (2018). Internacionalización de la educación superior en América Latina y el Caribe. Un balance. UNESCO-IESALC.

* Hernández, M. (1998). Políticas estatales en materia de evaluación. En Tres décadas de políticas del Estado en la educación superior (pp. 101-132). Asociación Nacional de Universidades e Instituciones de Educación Superior.

- Ley General de Educación Superior. (2021,20 de abril). Diario Oficial de la Federación. https://www.dof.gob.mx/nota_detalle.php?codigo=5616253\&fecha=20/04/2021

- Licandro, O. D. y Yepes Chisco, S. L. (2018). La Educación Superior conceptualizada como bien común: el desafío propuesto por unEsco. Revista Digital de Investigación en Docencia Universitaria, 12(1), 6-33. http://dx.doi.org/10.19083/ridu.12.715

* Marginson, S. (2016). Public/Private in higher education: a synthesis of economic and political approaches. Centre for Global Higher Education, 43(2), 322-337. http:// doi.org/10.1080/03075079.2016.1168797

* Mendoza Rojas, J. (2017). Financiamiento de la educación superior en la primera mitad del gobierno de Enrique Peña Nieto: ¿fin del periodo de expansión? Perfiles Educativos, 39 (156), 119-140. https://doi.org/10.22201/ iisue.24486167e.2017.156.58288 
* Moreno, C. y Muñoz. C. (2016). Innovación en el financiamiento de la educación superior en México. En Innovación de la educación superior. Perspectivas y nuevos retos (pp. 131-160). UdeG-UNESCO-IIPE.

* Murayama, C. (2009). La economía política de la educación superior en México. Asociación Nacional de Universidades e Instituciones de Educación Superior.

* Organización para la Cooperación y el Desarrollo Económico (OCDE). (2020). Panorama de la educación 2020. https://cutt.ly/fWVmnj7

* La educación como bien público [Editorial]. (2014). Revista Latinoamericana de estudios educativos, 44(4), 5-11. https://www.redalyc.org/pdf/270/27032872001. pdf

- Salmi, J. (2017). The Tertiary Education Imperative. Sense Publishers.

* Salmi, J. (2018). All around the world - Higher education equity policies across the globe. Fundación Lumina.

* Vallaeys, F. (2018). Las diez falacias de la Responsabilidad Social Universitaria. Revista digital de investigación en docencia universitaria, 12(1), 34-58. https://cutt. ly/tWZ1nZV

\section{Cómo citar este ARTículo}

* Fuentes Hernández, Cynthia Paola. (2021, septiembre-octubre). Ausencia de una política para el financiamiento de la educación superior. El caso mexicano. Revista Digital Universitaria (RDU), 22(5). http://doi.org/10.22201/ cuaieed.16076079e.2021.22.5.10 